\title{
Alpaca fiber growth is mediated by microRNA let-7b via down-regulation of target gene FGF5
}

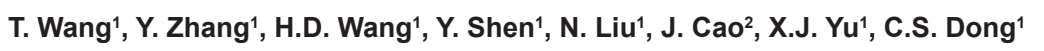
and X.Y. $\mathrm{He}^{1}$

${ }^{1}$ Alpaca Bioengineering Laboratory, College of Animal Science, Shanxi Agricultural University, Shanxi, China

${ }^{2}$ Beijing Vocational College of Agriculture, Beijing, China

Corresponding author: X.Y. He

E-mail: sxndhxy@163.com

Genet. Mol. Res. 14 (4): 13754-13763 (2015)

Received April 10, 2015

Accepted August 12, 2015

Published October 28, 2015

DOI http://dx.doi.org/10.4238/2015.October.28.38

ABSTRACT. MicroRNAs are very small endogenous RNA molecules that play a crucial role in an array of biological processes, including regulation of skin morphogenesis. The microRNA let-7b is thought to modulate animal hair growth, by binding target genes that encode growth factors. Fibroblast growth factor 5 (FGF5) has been previously reported to be involved in the initiation of the catagen phase of hair growth. In this study, we combined previous reports with bioinformatic analysis techniques to identify and validate $F G F 5$ and, using lucerifase assay, confirmed targeted binding of let-7b to FGF5. To investigate the interaction between let-7b and FGF5, alpaca skin fibroblasts were transfected with let-7b over-expression vectors, and then mRNA and protein expression levels of FGF5 and the gene encoding its receptor, FGFR1, were evaluated. Levels of FGF5 mRNA and protein were remarkably lower in transfected groups, as compared to controls. In summary, this study confirmed that let- $7 \mathrm{~b}$ acts as a regulator of skin morphogenesis, by directly targeting FGF5 and down-regulating its 
expression. It provides the evidence of hair growth regulated by miRNAs in animals and may have important applications in wool production.

Key words: Dual-luciferase reporter assay system; Alpaca fiber; Fibroblast growth factor 5; MicroRNA

\section{INTRODUCTION}

The alpaca is a valuable wool animal, and the yield and quality of alpaca fiber are therefore important production traits. In recent years, the regulatory mechanisms of alpaca fiber growth and coat color have aroused scientific interest, due to the excellent quality of alpaca wool (Dong et al., 2010; Fan et al., 2010; Zhu et al., 2010; Dong et al., 2012). It has been recently shown that microRNAs (miRNAs) play pivotal roles in skin morphogenesis and development, and are involved in a variety of skin diseases, including cancer (Sand et al., 2009). The physiological mechanism of hair growth is extremely complex requiring the coordinated regulation of many growth factors. MicroRNAs regulate animal hair growth by targeting growth factor genes (Ahmed et al., 2011; Yuan et al., 2013).

The hair follicle produces hair through a dynamic cycle of tissue growth and remodeling (Hardy, 1993). In mammals, the hair growth cycle consists of three defined phases: anagen, the phase during which the follicles grow and produce hair, and skin thickness increases; catagen, when the follicles regress and hair elongation ceases because the hair matrix cells stop proliferating; and telogen, at which point the hair is loosely anchored in a keratin matrix and follicles enter a quiescent phase (Paus and Cotsarelis, 1999); subsequently, a new cycle of hair growth is initiated. Previous reports have suggested that fibroblast growth factors (FGFs) are involved in the hair growth cycle. At least 22 members of the FGF family of genes have been discovered, and have various biologic activities (Abrahm et al., 1986). Among these, acidic FGF (FGF1) and basic FGF (FGF2) were the first two to be identified, and were named for their ability to stimulate fibroblast proliferation (Gospodarowicz, 1988). However, it is now known that FGFs can stimulate or inhibit proliferation of various cell types, and therefore play a role in extensive developmental processes, including hair follicle growth (Klagsbrun, 1989). The gene FGF5 was originally reported as a human oncogene (Zhan et al., 1987), but was categorized into the FGF family due to its high homology with acidic and basic FGF (Haub and Goldfarb, 1991). In mice, animals with a recessive phenotype, known as angora(go), have an anagen VI phase(new hair shaft emerges from skin surface) that is approximately $50 \%$ longer than this phase of hair development in heterozygotes or wild-type mice. This abnormally long anagen VI results in the production of hair that is about $50 \%$ longer (Pennycuik and Raphael, 1984). Subsequent research identified a null mutation in FGF5 resulted in the angora phenotype, indicating that the transition between anagen and catagen is normally regulated by FGF5 (Baird and Klagsbrun, 1991). FGF5 exerts a significant impact on the initiation of catagen; FGF5 expression is initiated in the outer root sheath after follicles enter anagen VI, and is down-regulated just prior to the onset of catagen (Goldfarb, 1990). A high-affinity receptortyrosine kinase is required in FGF signal transduction for ligand binding, and numerous in vitro studies have provided evidence of high affinity binding of FGF5 to FGFR1 (Fibroblast growth factor receptor 1) (Ornitz and Leder, 1992).

MicroRNAs are small RNA molecules, approximately 21-25 nucleotides (nt) in length, and are essential regulators of a wide range of cellular processes (Chen et al., 2006). Research have shown that skin morphogenesis requires highly coordinated, undisrupted miRNA metabolism. High expression levels of several miRNAs in the epidermis and hair follicles are necessary 
for normal skin development (Yi et al., 2006). The miRNA let-7 was originally discovered as a switch gene in Caenorhabditis elegans (Reinhart et al., 2000). Let-7 family members have been implicated as tumor suppressors and are commonly down-regulated in many human cancers (Akao et al., 2006). Lai (2002) reported that miRNAs mediate gene post-transcriptional regulation through binding messenger RNA (mRNA) with the first eight sequences of miRNA, known as seed sequence. The mature sequences of alpaca let-7b have been previously reported as: TGAGGTAGTAGGTTGTGTGGTT (Tian et al., 2012).

We previously analyzed the miRNA expression profile of alpaca skin, using miRNA microarray and real-time polymerase chain reaction (PCR) technology, and identified 39 miRNAs (including let-7b) with significantly different levels of expression in skin of the ear and back (He et al., 2010). Combining previous reports with bioinformatics prediction analysis to identify potential target genes for identified miRNAs, we described FGF5 as a predicted target gene for let-7b. A further study investigated differences in FGF5 expression levels in alpaca skin from the ear and back, and showed that correlative miRNA expression level from the ear and back of alpaca skin were in perfect accordance with FGF5 expression levels. (Liu et al., 2011).

In the current study, dual-luciferase assays were performed to test whether let-7b directly targets FGF5 through the 3'-untranslated region (UTR). We then used alpaca fibroblasts to determine whether let-7b down-regulated FGF5 at the mRNA or protein level, or both.

\section{MATERIAL AND METHODS}

\section{Cell culture}

Alpaca fibroblasts were maintained in FM (Fibroblast Medium) (Thermo,MA, USA) 293T cells were grown in DMEM (Dulbecco Modified Eagle Medium) high glucose medium (Thermo, MA, USA) with $10 \%$ fetal bovine serum and $100 \mu \mathrm{g} / \mathrm{mL}$ penicillin and streptomycin (Invitrogen, Beijing, China).

\section{Plasmids}

Fragments (665 bp) of FGF5 3'-UTR were amplified by PCR using the primers FGF 3'-UTR forward (5'-GAGCTCGACTTACTTTGGTTTTGTGAGA-3') and reverse (5'-GAATTCCTGTCGTCT GCACTTCCTGT-3'). Fragments were then inserted into the pmirGLO dual-luciferase miRNA target expression vector (Promega, Madison, WI, USA). Plasmids were verified by sequencing. The pcDNA6.2 ${ }^{\mathrm{TM}}-\mathrm{GW} /$ EmGFP-miR vector was purchased from Invitrogen TM, Life technologies company, and the construction of the let-7b over-expression and negative control vectors was supported by Life technologies. The oligo DNAs inserted into the expression vector for the negative control and let-7b were as follows: oligo DNA sequence 5'-3'; miRNA mature sequence: UGAGGUA GUAGGUUGUGUGGUU; negative control-F: GCGTGGAGACGTTTTGGCCACTGACTGACGTC TCCACGCAGTACATTT; negative control-R: TGGAGACGTCAGTCAGTGGCCAAAACGTCTCCA CGCGCAGTACATTTc; Let-7b vector-F: TGTGTGGTTGTTTTGGCCACTGACTGACAACCACAC CCTACTACCTCA; Let-7b vector-R: TGGTTGTCAGTCAGTGGCCAAAACAACCACACAACCTACT ACCTCAC.

\section{Luciferase reporter assay}

Reporter plasmid was co-transfected, with let-7b over-expression vector or negative 
control vector, into HEK293 cells seeded in a 24-well plate. Dual-luciferase assay was conducted at $48 \mathrm{~h}$ following transfection, using the manufacturer's protocol (Attractene Transfection reagent, Qiagen, Shanghai,China). Renilla and firefly luciferase served as control and experimental reporters, respectively. Renilla and firefly luciferase activities were measured with a GLoMax 96 microplate luminometer (Promega, Madison, WI, USA)), and ratios were normalized to the negative control and further normalized to an empty plasmid control. Three independent experiments were conducted.

\section{RNA isolation, reverse transcription and real-time PCR}

Alpaca fibroblasts were transfected with let-7b over-expression vector. At $48 \mathrm{~h}$ after transfection, total RNA from untransfected and transfected cells was isolated using Trizol reagent (Invitrogen, Beijing, China). Concentrations of RNA were determined by spectrophotometer (Nanodrop-1000, Thermo,MA, USA). For quantitation of FGF5 and FGFR1, reverse transcription and PCR were performed using PrimeScript RT master mix (TAKARA, Dalian, China) and QuantiFast SYBR green PCR (Qiagen, Shanghai, China) kits, according to manufacturer instructions. Briefly, RNA (40 ng) was reverse transcribed in a $15 \mu \mathrm{L}$ reaction using specific primers for FGF5 and FGFR1. CDNA $(2 \mu \mathrm{L})$ was used for the PCR amplification in a $20 \mu \mathrm{L}$ reaction using the 7500 Fast real-time PCR system (Life technologies, MA, USA). For each sample, PCR was performed in triplicate. $\beta$-actin was used as an endogenous control for normalization of target gene expression, and differences between samples and controls were calculated using the $2^{-\triangle \Delta \mathrm{Ct}}$ method.

\section{Western blotting (WB)}

Cells were lysed using cell lysis buffer (Beyotime, Beijing, China) and protein concentrations were determined using a spectrophotometer (Nanodrop-1000, Thermo). Total protein (10 $\mu \mathrm{g}$ ) was separated on a $12 \%$ polyacrylamide (w/v) gel, and electro-transferred onto Immobilon-PSQ PVDF transfer membrane (0.45 um; Millipore, Massachusetts, USA). Following blocking with 5\% nonfat milk (w/v) in TBS-T (Tris-buffered saline with $0.1 \%$ Tween-20), membranes were incubated at $4^{\circ} \mathrm{C}$ overnight with rabbit polyclonal antibody against FGF5 (diluted 1:500) and rabbit polyclonal antibody against FGFR1 (diluted 1:300; both Proteintech, Wuhan, China), followed by washing three times for 10 min each in TBS-T. Membranes were incubated with horseradish peroxidaseconjugated goat anti-rabbit IgG secondary antibody (CWBIO, Beijing, China) at room temperature for $1 \mathrm{~h}$, followed by washing three times for $10 \mathrm{~min}$ each with TBS-T. Immunoreactive bands were visualized using an enhanced chemiluminescence (ECL) kit (CWBIO, Beijing, China).

\section{Immunocytochemistry (ICC)}

Alpaca fibroblasts were plated on slides and cultured until they reached $80-90 \%$ confluency within 24 and $48 \mathrm{~h}$, and then processed for ICC using a previously described protocol (Cekanova et al., 2013). Cells were fixed with $4 \%$ paraformaldehyde for $30 \mathrm{~min}$ at room temperature and blocked for $30 \mathrm{~min}$ in protein block solution. Cells were then were incubated with FGF5 rabbit polyclonal antibody (Proteintech, Wuhan, China), then incubated with specific secondary antibodies, conjugated with horseradish peroxidase (CWBIO, Beijing, China), for 45 min at $37^{\circ} \mathrm{C}$. Following washing 3 times ( 5 min each) in PBS, immunoreactivity was visualized in the presence of 3,3-diaminobenzidine (DAB, Tiangen Biotechnology Co., Beijing, China) substrate. Cell nuclei were counter-stained with hematoxylin, and slides were mounted, cover-slipped, and evaluated under a 
Leitz DMRB microscope (Leica, Solms, Germany). For negative controls, slides were processed as outlined above, except that the primary antibody was replaced by non-immune rabbit serum.

\section{RESULTS}

\section{Let-7b directly targets FGF5}

In previous studies by our research group, let-7b appeared to be involved in hair growth regulation, and had differential expression in alpaca skin from parts of the body with different fiber qualities (Liu et al., 2011). Bioinformatics predictions in publicly available algorithms (TargetScan4.1, www.targetscan.org; miRBase) indicated that FGF5 might be targeted by let-7b. Figure 1 shows that the let-7b binding site is located within the 3'-UTR of FGF5. In combination with target prediction algorithms, we constructed a dual-luciferase recombinant vector containing the 3'-UTR of FGF5. 293T cells were co-transfected with the let-7b over-expression vector and dual-luciferase reporter plasmid for FGF5, and dual-luciferase assays were performed. Luciferase activity was decreased to $34 \%$, normalized firefly luciferase activity (firefly luciferase activity/Renilla luciferase activity) for each construct was compared to that of the pmirGLO Vector no insert control, (Figure 2). This observation provided direct evidence of let-7b targeting of FGF5. We therefore concluded that FGF5 is directly regulated by let-7b.

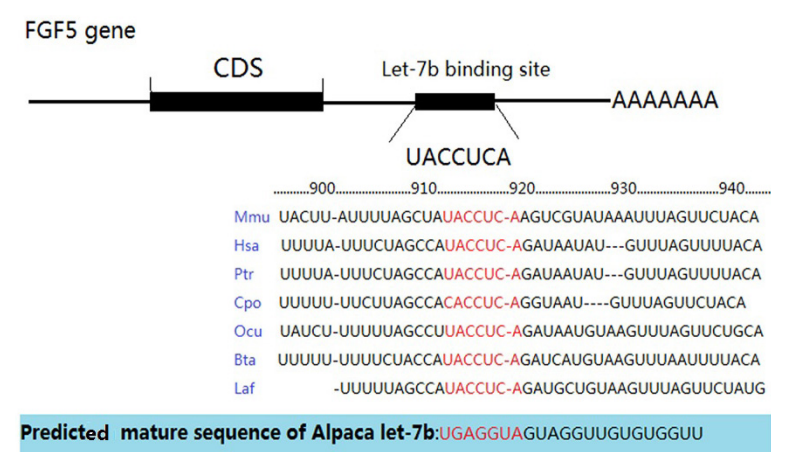

Figure 1. Schematic diagram of let-7b target binding site in 3'-UTR of FGF5, and predicted mature sequence of alpaca let-7b. Mmu, mouse; Hsa, human; Ptr, chimpanzee; Cpo, guinea pig; Ocu, rabbit; Bta, cow; Laf, elephant.

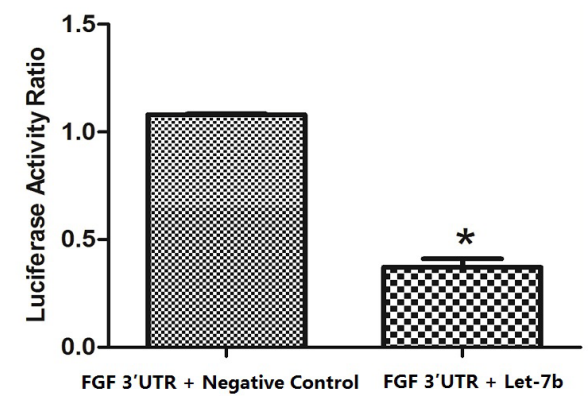

Figure 2. Relative luciferase activity following co-transfection of let-7b over-expression vector and dual-luciferase reporter plasmid for FGF5 3'-UTR. Luciferase activity of the reporter gene containing wild type 3'-UTR of FGF5 was significantly suppressed in the let-7b vector group, compared with the control. Asterisk indicates significant difference $(P<0.05)$. In the control group, "negative control" indicates the negative control expression vector. 


\section{Immunolocalization and cellular expression of FGF5}

Alpaca fibroblasts were transfected with either the let-7b over-expression or negative control vectors. As shown in Figure 3 (A-D), positive reactivity was noted as predominantly fibroblastic cytoplasmic staining. Negative controls showed no specific immunostaining of fibroblasts (Figure 3, E and F). FGF5 immunoreactivity was clearly observed in fibroblast cytoplasm in the negative control vector-transfected group (Figure 3, B and D). In contrast, immunoreactivity staining was barely detected in the let-7b over-expression vector-transfected group (Figure 3, A and C), which was consistent with FGF5 mRNA and protein expression level results.

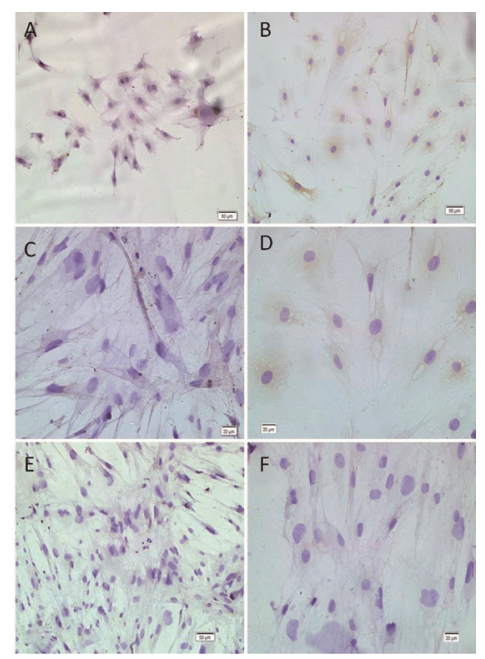

Figure 3. Immunohistochemistry results of FGF5 expression. Positive reactivity was noted predominantly as fibroblast cytoplasmic staining (A-D). Fibroblasts in negative control groups showed no specific immunostaining (E and F). FGF5 immunoreactivity was clearly observed in fibroblastic cytoplasm in the negative control vector-transfection group (B and $\mathbf{D}$ ). Immunoreactivity staining was barely detected in the let-7b over-expression vector-transfection group (A and C), compared with the negative control group.

\section{Let-7b down-regulates FGF5 at the mRNA and protein level}

To further evaluate let-7b regulation of FGF5, alpaca fibroblasts were transfected with let$7 \mathrm{~b}$ over-expression or negative control vectors, and cultured under standard conditions. As shown in Figure 4, different fibroblast morphology was noted between the let-7b vector-transfected and the nontransfected group; cells in the transfected group were in poor growth condition, suggesting that the let-7b over-expression vector may exert a toxic effect on cells. We performed quantitative real-time PCR and western blot analysis to detect mRNA and protein expression levels of FGF5 and FGFR1. As shown in Figure 5 and Figure 6, over-expression of let-7b decreased expression levels of FGF5 mRNA and protein, but indirectly increased both mRNA and protein expression levels of FGFR1. In the transfected groups, FGF5 mRNA quantity was 0.287 times that of the control groups; FGFR1 mRNA quantity was 2.251 times that of the control groups $(P<0.01)$. Protein levels of FGF5 in the transfected groups were significantly lower than for control groups $(P$ $<0.05)$. The average relative gray values of FGF5 and FGFR1 proteins were $0.224 \pm 0.019$ and $0.952 \pm 0.152$, respectively. 


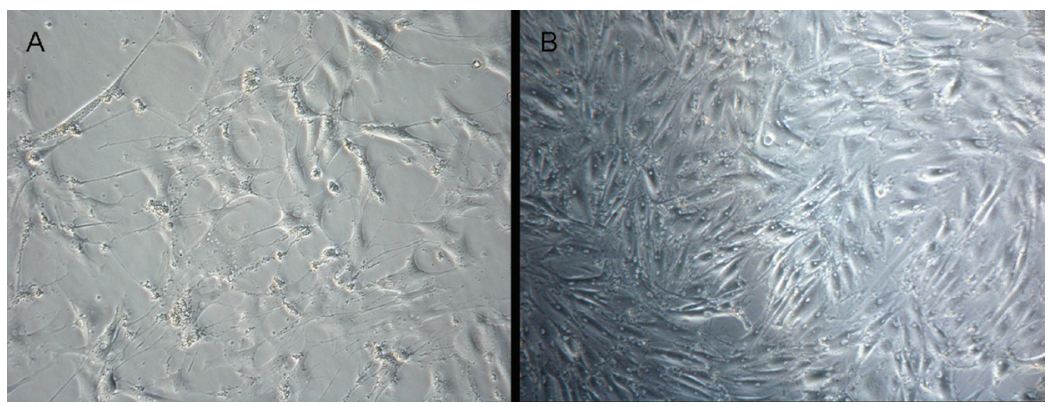

Figure 4. Morphology of fibroblasts transfected with let-7b vector. By optical microscope; fibroblasts following (A) and prior to (B) transfection (both 20X objective).
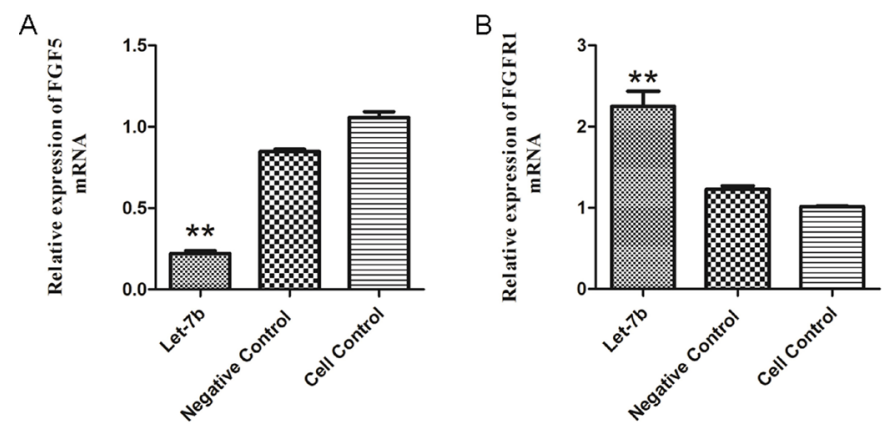

Figure 5. Effects of let-7b on the expression of FGF5 and FGFR1 in fibroblasts. By quantitative real-time PCR. A. Expression of FGF5 mRNA in different groups. Over-expression let-7b significantly decreased FGF5 mRNA expression; B. expression of FGFR1 mRNA in different groups. Data are presented as the mean \pm SD (error bars). Double asterisks indicate significant difference $(P<0.01)$.

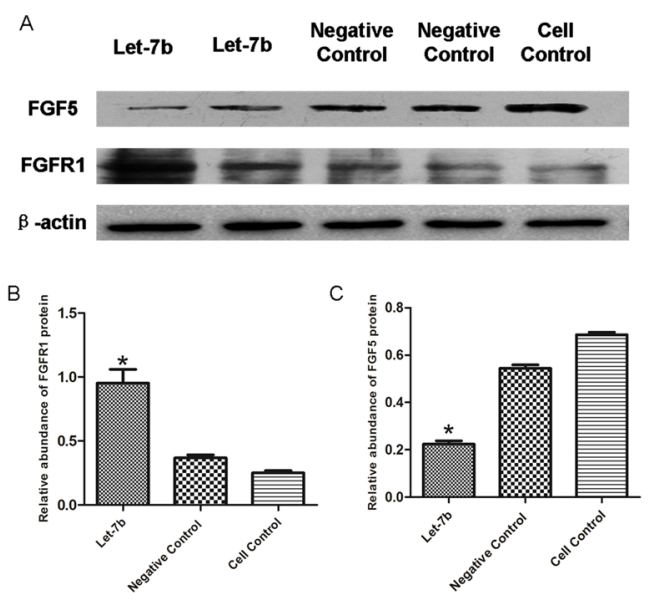

Figure 6. Relative abundance of FGF5 and FGFR1 proteins. By immunoblotting analysis. Following transfection for $48 \mathrm{~h}$, protein bands of FGF5 and FGFR1 can be seen. A. Band intensities corresponding to FGF5 were compared to those corresponding to $\beta$-actin. B. Relative abundance of FGFR1 was determined by analyzing band intensities. C. Band intensities were quantified by Image J. Standard line represents mean \pm SD. Asterisk indicates significant difference $(P<0.05)$. 


\section{DISCUSSION}

A new strategy of gene regulation was defined by the activities of Caenorhabditis elegans miRNAs let-7b and lin-4. These RNA molecules of 21-22 nt are complementary to the 3'-UTRs of target transcripts, and mediate negative post-transcriptional regulation through RNA duplex formation (Wightman et al., 1993; Lee et al., 1993; Ha et al., 1996; Reinhart et al., 2000). Let-7 and its family members are highly conserved across species in sequence and function, and have various biological effects, including development of the nervous system and liver, and the inhibition of cell proliferation and the invasion of cancer cells (Johnson et al., 2007; Zhang et al., 2007; Maller Schulman et al., 2008).

The texture and quality of alpaca fiber from different parts of the body is not the same; for instance, the ear hair is shorter than hair from the back, and has a shorter growth cycle. We previously observed that let-7b is expressed at higher levels in alpaca skin on the back, compared to the ear, suggesting that let-7b may mediate the hair growth process through regulating target genes involved in hair follicle development (He et al., 2010; Liu et al., 2011). Based on this, we screened for predicted targets and identified FGF5 as a plausible candidate. In the current study, dual-luciferase assay was performed to validate that let-7b can efficiently target FGF5 3'-UTR. The classical mechanism of let-7 action involves its binding to the 3'-UTR of target mRNAs in order to regulate their expression. However, a previous report suggested that let-7a caused identical responses when it was targeted to the 5'- or 3'-UTRs of mRNAs containing internal ribosome entry sites (Lytle et al., 2007), indicating that let-7 is able to act through binding to sites other than the 3'-UTR of target mRNAs. In the current study, successful construction of a dual-luciferase reporter vector containing FGF5 3'-UTR demonstrated that let-7b might regulate target genes expression through the classical mechanism. We have confirmed the target relationship between let-7b and FGF5, and further validated down-regulation of FGF5 at the protein and mRNA level by let-7b, providing a theoretical basis for ongoing study of this interaction.

Hair is produced in a cycle of tissue growth, degeneration and renewal which is involved in a series of factor effects, among them FGFs play an important role in hair growth. Rosenquist and Martin (1996) demonstrated that FGF1 is expressed in the keratogenous zone and the inner root sheath higher up in the follicle neck, FGF2 is localized to the basement membrane of the outer root sheath and hair matrix, and only FGF7 is expressed in the hair follicle. In spite of a deletion of FGF5 in mice with the recessive angora mutation, transition between the anagen and catagen phases of hair follicle growth still occurs (Ito et al., 2003). The protein, FGF5, has been shown to bind to FGFR1 with high affinity; furthermore, FGF5 and FGF2 share the same receptor (FGFR1c) on NIH/3T3 cells (Cekanova et al., 2003). In the in vitro study reported here, when FGF5 was down-regulated by over-expression of let-7b in alpaca fibroblasts, the expression of FGFR1 was elevated; the reason for this is not clear. It is possible that other members of the FGF family compensate for a reduced effect of FGF5 (FGF2 may be a potential candidate) and that increased FGFR1 expression may be associated with binding with FGF2. Further investigation, however, is needed to confirm this. Previous studies have suggested that FGFs may have roles as key regulators in the maintenance of normal vasculature (Hatanaka et al., 2011). Fibroblast growth factors regulate TGF- $\beta$ (Transforming growth factor- $\beta$ ) signaling involved in endothelial-to-mesenchymal transition, by controlling let-7 miRNA expression (Chen et al., 2012). Previous studies indicated that let-7 is a pivotal factor to connect the two signaling pathways (FGF signaling and TGF- $\beta$ signaling). The effect of the interaction between let-7b and FGF5 and FGFR1 on hair growth is only one component of the myriad biological effects of let-7b; further studies are warranted to further explore the role of this miRNA. 


\section{ACKNOWLEDGMENTS}

Research supported by grants from the National Natural Science Foundation of China (grant \#31172283 and \#31302049); Shanxi Agricultural University Research startup project for introducing talents, "2009", (grant \#XB2009020); and Shanxi Science and Technology Issue Foundation (grant \#20080311034).

\section{REFERENCES}

Abrahm JA, Whang JL, Tumolo A, Mergia A, et al. (1986). Human basic fibroblast growth factor: nucleotide sequence and genomic organization. EMBO J. 5: 2523-2528.

Ahmed MI, Mardaryev AN, Lewis CJ, Sharov AA, et al. (2011). MicroRNA-21 is an important downstream component of BMP signaling in epidermal keratinocytes. J. Cell Sci. 124: 3399-3404.

Akao Y, Nakagawa $Y$ and Naoe T (2006). Let-7 microRNA functions as a potential growth suppressor in human colon cancer cells. Biol. Pharm. Bull. 29: 903-906.

Baird A and Klagsbrun M (1991). The fibroblast growth factor family. Cancer Cells 3: 239-243.

Cekanova M, Uddin MJ, Bartges JW, Callens A, et al. (2013). Molecular imaging of cyclooxygenase-2 in canine transitional cell carcinoma as in vitro and in vivo. Cancer Prev. Res. (Phila). 6: 466-476.

Chen CH, Gou M and Hay BA (2006). Identifying microRNA regulators of cell death in Drosophila. Methods Mol. Biol. 342: 229-240.

Chen PY, Qin L, Barnes C, Charisse K, et al. (2012). FGF regulates TGF- $\beta$ signaling and endothelial-to-mesenchymal transition via control of let-7 miRNA expression. Cell Rep. 2: 1684-1696.

Dong CS, Wang H, Xue L, Dong Y, et al. (2012). Coat color determination by miR-137 mediated down-regulation of microphthalmia-associated transcription factor in a mouse model. RNA 18: 1679-1686.

Dong Y, Cao J, Wang H, Zhang J, et al. (2010). Nitric oxide enhances the sensitivity of alpaca melanocytes to respond to alpha-melanocyte- stimulating hormone by up-regulating melanocortin-1 receptor. Biochem. Biophys. Res. Commun. 396: 849-853.

Fan RW, Yang G and Dong CS (2010). Study of hair melanins in various hair color alpaca (Lama pacos). Asian Australas. J. Anim. Sci. 4: 444-449.

Goldfarb M (1990). The fibroblast growth factor family. Cell Growth Differ. 1: 439-445.

Gospodarowicz D (1988). Molecular and developmental biology aspects of fibroblast growth factor. Adv. Exp. Med. Biol. 234: 23-39.

Ha I, Wightman B and Ruvkun G (1996). A bulged lin-4/lin-14 RNA duplex is sufficient for Caenorhabditis elegans lin-14 temporal gradient formation. Genes Dev. 10: 3041-3050.

Hardy MH (1993). The secret life of the hair follicle. Trends Genet. 8: 55-61.

Hatanaka K, Simons M and Murakami M (2011). Phosphorylation of VE-cadherin controls endothelial phenotypes via p120catenin coupling and Rac1 activation. Am. J. Physiol. Heart Circ. Physiol. 300: H162-172.

Haub O and Goldfarb M (1991). Expression of the fibroblast growth factor-5 gene in the mouse embryo. Development 112: $397-406$.

He XY, Hao HQ, Liu DD, et al. (2010). Identification of differential expression microRNAs in differential areas of skin in the adult alpaca. Chin. J. Bioch. Mol. Biol.11: 1016-1022.

Ito C, Saitoh Y, Fujita Y, Yamazaki Y, et al. (2003). Decapeptide with fibroblast growth factor (FGF)-5 partial sequence inhibits hair growth suppressing activity of FGF-5. J. Cell. Physiol. 197: 272-283.

Johnson CD, Esquela-Kerscher A, Stefani G, Byrom M, et al. (2007). The let-7 microRNA represses cell proliferation pathways in human cells. Cancer Res. 67: 7713-7722.

Klagsbrun M (1989). The fibroblast growth factor family: structural and biological properties. Prog. Growth Factor Res. 1: 207-235.

Lai EC (2002). MicroRNAs are complementary to 3'-UTR sequence motifs that mediate negative post-transcriptional regulation. Nat. Genet. 30: 363.

Lee RC, Feinbaum RL and Ambros V (1993). The C. elegans heterochronic gene lin-4 encodes small RNAs with antisense complementarity to lin-14. Cell 75: 843-854.

Liu DD, Zhang ZY, He XY, Dong YJ, et al. (2011). FGF5 expression and immunolocalization in back and ear skin of young alpaca (Lama pacos). Chin. J. Bioch. Mol. Biol. 5: 473-479.

Lytle JR, Yario TA and Steitz JA (2007). Target mRNAs are repressed as efficiently by microRNA-binding sites in the 5'-UTR as in the 3'-UTR. Proc. Natl. Acad. Sci. U.S.A. 104: 9667-9672.

Maller Schulman BR, Liang X, Stahlhut C, DelConte C, et al. (2008). The let-7 microRNA target gene, Mlin41/Trim71 is required for mouse embryonic survival and neural tube closure. Cell Cycle 7: 3935-3942. 
Ornitz DM and Leder P (1992). Ligand specificity and heparin dependence of fibroblast growth factor receptors 1 and 3. J. Biol. Chem. 267: 16305-16311.

Paus R and Cotsarelis G (1999). The biology of hair follicles. N. Engl. J. Med. 341: 491-497.

Pennycuik PR and Raphael KA (1984). The angora locus (go) in the mouse: Hair morphology, duration of growth cycle and site of action. Genet. Res. 44: 283-291.

Reinhart BJ, Slack FJ, Basson M, Pasquinelli AE, et al. (2000). The 21-nucleotide let-7 RNA regulates developmental timing in Caenorhabditis elegans. Nature 403: 901-906.

Rosenquist TA and Martin GR (1996). Fibroblast growth factor signalling in the hair growth cycle: expression of the fibroblast growth factor receptor and ligand genes in the murine hair follicle. Dev. Dyn. 205: 379-386.

Sand M, Gambichler T, Sand D, Skrygan M, et al. (2009). Micro RNAs and the skin: tiny players in the body's largest organ. J. Dermatol. Sci. 53: 169-175.

Tian X, Jiang J, Fan R, Wang H, et al. (2012). Identification and characterization of microRNAs in white and brown alpaca skin. BMC Genomics 13: 555.

Wightman B, Ha I and Ruvkun G (1993). Posttranscriptional regulation of the heterochronic gene lin-14 by lin-4 mediates temporal pattern formation in C. elegans. Cell 75: 855-862.

Yi R, O'Carroll D, Pasolli HA, Zhang Z, et al. (2006). Morphogenesis in skin is governed by discrete sets of differentially expressed microRNAs. Nat. Genet. 38: 356-362.

Yuan C, Wang X, Geng R, He X, et al. (2013). Discovery of cashmere goat (Capra hircus) microRNAs in skin and hair follicles by Solexa sequencing. BMC Genomics 14: 511.

Zhan X, Culpepper A, Reddy M, Loveless L, et al. (1987). Human oncogenes detected by a defined medium culture assay. Oncogene 1: 369-376.

Zhang B, Pan X, Cobb GP and Anderson TA (2007). MicroRNAs as oncogenes and tumor suppressors. Dev. Biol. $302:$ 1-12.

Zhu Z, He J, Jia X, Jiang J, et al. (2010). MicroRNA-25 functions in regulation of pigmentation by targeting the transcription factor MITF in alpaca (Lama pacos) skin melanocytes. Domest. Anim. Endocrinol. 38: 200-209. 